\title{
Publisher Correction: The toughness of mechanical metamaterials
}

Angkur Jyoti Dipanka Shaikeea (D), Huachen Cui (D), Mark O'Masta (D), Xiaoyu Rayne Zheng (1) and Vikram Sudhir Deshpande (i)

Correction to: Nature Materials https://doi.org/10.1038/s41563-021-01182-1, published online 7 February 2022

In the version of this article initially published, Xiaoyu Rayne Zheng (rayne@seas.ucla.edu) was not listed as a co-corresponding author. The error has been corrected in the HTML and PDF versions of the article.

Published online: 16 February 2022

https://doi.org/10.1038/s41563-022-01217-1

(c) The Author(s), under exclusive licence to Springer Nature Limited 2022 